Alexandru Dimca $\cdot$ Alexander I. Suciu

\title{
Which 3-manifold groups are Kähler groups?
}

Received November 6, 2007 and in revised form May 17, 2008

Abstract. The question in the title, first raised by Goldman and Donaldson, was partially answered by Reznikov. We give a complete answer, as follows: if $G$ can be realized as both the fundamental group of a closed 3-manifold and of a compact Kähler manifold, then $G$ must be finite-and thus belongs to the well-known list of finite subgroups of O(4), acting freely on $S^{3}$.

Keywords. Kähler manifold, 3-manifold, fundamental group, cohomology ring, resonance variety, isotropic subspace

\section{Introduction}

1.1. As is well-known, every finitely presented group $G$ occurs as the fundamental group of a smooth, compact, connected, orientable 4-dimensional manifold $M$. As shown by Gompf [14], the manifold $M$ can be chosen to be symplectic. Requiring a complex structure on $M$ is no more restrictive, as long as one is willing to go up to complex dimension 3 (see Taubes [32]).

Suppose now $G$ is the fundamental group of a compact Kähler manifold $M$. Groups arising this way are called Kähler groups (or, projective groups, if $M$ is actually a smooth projective variety). The Kähler condition puts strong restrictions on what $G$ can be. For instance, the first Betti number, $b_{1}(G)$, must be even, by classical Hodge theory. Moreover, $G$ must be 1-formal, by work of Deligne, Griffiths, Morgan, and Sullivan [9]. Also, $G$ cannot split non-trivially as a free product, by a result of Gromov [17]. On the other hand, every finite group is a projective group, by a classical result of Serre [29]. We refer to [1] for a comprehensive survey of Kähler groups, and to the recent work of Delzant-Gromov [11], Napier-Ramachandran [25], and Delzant [10] for further geometric restrictions imposed by the Kähler condition on a group $G$.

A. Dimca: Laboratoire J. A. Dieudonné, UMR du CNRS 6621, Université de Nice-Sophia Antipolis, Parc Valrose, 06108 Nice Cedex 02, France; e-mail: dimca@ math.unice.fr

A. Suciu: Department of Mathematics, Northeastern University, Boston, MA 02115, USA; e-mail: a.suciu@neu.edu

Mathematics Subject Classification (2000): Primary 20F34, 32J27, 57N10; Secondary 14F35, $55 \mathrm{~N} 25$ 
Requiring that $M$ be a 3-dimensional compact, connected manifold also puts severe restrictions on $G=\pi_{1}(M)$. For example, if $G$ is abelian, then $G$ is either $\mathbb{Z} / n \mathbb{Z}, \mathbb{Z}$, $\mathbb{Z} \oplus \mathbb{Z}_{2}$, or $\mathbb{Z}^{3}$ (see $[20]$ ).

1.2. A natural question - raised by Goldman and Donaldson in 1989, and independently by Reznikov in 1993 - is then: what are the 3-manifold groups which are Kähler groups?

In [28], Reznikov proved the following result, which Simpson [31] calls "one of the deepest restrictions" on the homotopy types that may occur for Kähler manifolds: Let $M$ be an irreducible, atoroidal 3-manifold, and suppose there is a homomorphism $\rho: \pi_{1}(M) \rightarrow \operatorname{SL}(2, \mathbb{C})$ with Zariski dense image. Then $G=\pi_{1}(M)$ is not a Kähler group. The same conclusion was reached by Hernández-Lamoneda in [19], under the assumption that $M$ is a geometrizable 3-manifold, with all pieces hyperbolic.

In this note, we answer the above question for all 3-manifold groups, as follows.

Theorem 1.1. Let $G$ be the fundamental group of a compact, connected 3-manifold. If $G$ is a Kähler group, then $G$ is finite.

By the 3-dimensional spherical space-form conjecture, now established by Perelman [26, 27], a closed 3-manifold $M$ has finite fundamental group if and only if it admits a metric of constant positive curvature (for a detailed proof, see Morgan and Tian [24, Corollary 0.2]). Thus, $M=S^{3} / G$, where $G$ is a finite subgroup of $\mathrm{O}(4)$, acting freely on $S^{3}$. The list of such finite groups (essentially due to Hopf) is given by Milnor in [23].

1.3. The paper is organized as follows. In $\$ 2$, we discuss the characteristic and resonance varieties of a group $G$, and two notions of isotropy. In $\$ 3$, we recall the Isotropic Subspace Theorem of Catanese, and a correspondence due to Beauville. In \$4 we use these tools to prove a key result, tying the first resonance variety of a Kähler manifold to the rank of the cup-product map in low degrees. In $\$ 5$, we investigate the first resonance variety of a closed, oriented 3-manifold; Poincaré duality and properties of Pfaffians yield a very different conclusion in this setting.

All this works quite well, provided the first Betti number of $G$ is positive. To deal with the remaining case, we need two theorems of Reznikov and Fujiwara, relating the Kähler, respectively the 3-manifold condition on a group to Kazhdan's property $T$; we recall those in $\$ 6$. Finally, we put everything together in $\$ 7$, and give a proof of Theorem 1.1 .

A natural question arises out of this work: Which 3-manifold groups are quasi-Kähler? (A group $G$ is quasi-Kähler if $G=\pi_{1}(M \backslash D)$, where $M$ is a compact Kähler manifold and $D$ is a divisor with normal crossings.) We have some partial results in this direction; those results will be presented elsewhere.

\section{Cohomology jumping loci and isotropic subspaces}

2.1. Let $X$ be a connected CW-complex with finitely many cells in each dimension. Let $G=\pi_{1}(X)$ be the fundamental group of $X$, and $\mathbb{T}=\operatorname{Hom}\left(G, \mathbb{C}^{*}\right)$ its character variety. 
Every character $\rho \in \mathbb{T}$ determines a rank 1 local system, $\mathbb{C}_{\rho}$, on $X$. The characteristic varieties of $X$ are the jumping loci for cohomology with coefficients in such local systems:

$$
V_{d}^{i}(X)=\left\{\rho \in \mathbb{T} \mid \operatorname{dim} H^{i}\left(X, \mathbb{C}_{\rho}\right) \geq d\right\}
$$

The varieties $V_{d}(X)=V_{d}^{1}(X)$ depend only on $G=\pi_{1}(X)$, so we sometimes denote them as $V_{d}(G)$.

2.2. Consider now the cohomology algebra $A=H^{*}(X, \mathbb{C})$. Left multiplication by an element $x \in A^{1}$ yields a cochain complex $(A, x): A^{0} \stackrel{x}{\rightarrow} A^{1} \stackrel{x}{\rightarrow} A^{2} \rightarrow \cdots$. The resonance varieties of $X$ are the jumping loci for the homology of this complex:

$$
R_{d}^{i}(X)=\left\{x \in A^{1} \mid \operatorname{dim} H^{i}(A, x) \geq d\right\} .
$$

The varieties $R_{d}(X)=R_{d}^{1}(X)$ depend only on $G=\pi_{1}(X)$, so we sometimes denote them by $R_{d}(G)$. By definition, an element $x \in A^{1}$ belongs to $R_{d}(X)$ if and only if there exists a subspace $W \subset A^{1}$ of dimension $d+1$ such that $x \cup y=0$ for all $y \in W$.

Fix bases $\left\{e_{1}, \ldots, e_{n}\right\}$ for $A^{1}$ and $\left\{f_{1}, \ldots, f_{m}\right\}$ for $A^{2}$. Writing the cup-product as $e_{i} \cup e_{j}=\sum_{k=1}^{m} \mu_{i, j, k} f_{k}$, we may define an $m \times n$ matrix $\Delta$ of linear forms in variables $x_{1}, \ldots, x_{n}$, with entries

$$
\Delta_{k, j}=\sum_{i=1}^{n} \mu_{i, j, k} x_{i} .
$$

It is readily seen that $R_{d}(X)=V\left(E_{d}(\Delta)\right)$, where $E_{d}$ denotes the ideal of $(n-d) \times(n-d)$ minors. Note also that $x \cup x=0$ for all $x \in A^{1}$ implies $\Delta \cdot \vec{x}=0$, where $\vec{x}$ is the column vector with entries $x_{1}, \ldots, x_{n}$.

2.3. Foundational results on the structure of the cohomology support loci for local systems on compact Kähler manifolds were obtained by Beauville [2], Green-Lazarsfeld [15], Simpson [30], and Campana [5]: if $G$ is the fundamental group of such a manifold, then $V_{d}(G)$ is a union of (possibly translated) subtori of the algebraic group $\mathbb{T}$.

In addition, Theorem A from [12] establishes a strong relationship between the characteristic and resonance varieties of a Kähler group $G$ : the tangent cone to $V_{d}(G)$ at the identity of $\mathbb{T}$ equals $R_{d}(G)$ for all $d \geq 1$.

2.4. A non-zero subspace $E \subset H^{1}(X, \mathbb{C})$ is (totally) isotropic if the restriction of the cup-product map $\cup_{X}: H^{1}(X, \mathbb{C}) \wedge H^{1}(X, \mathbb{C}) \rightarrow H^{2}(X, \mathbb{C})$ to $E \wedge E$ is identically zero. By analogy, we say $E$ is 1-isotropic if the restriction of $\cup_{X}$ to $E \wedge E$ has 1-dimensional image.

Note that these properties of $E$ depend only on $G=\pi_{1}(X)$. Indeed, let $h: X \rightarrow$ $K(G, 1)$ be a classifying map. Then $h_{*}: H_{1}(X, \mathbb{Z}) \rightarrow H_{1}(G, \mathbb{Z})$ is an isomorphism, and $h_{*}: H_{2}(X, \mathbb{Z}) \rightarrow H_{2}(G, \mathbb{Z})$ is an epimorphism. Using Kronecker duality and the functoriality of the cup-product, it is readily seen that $E$ is a (1-) isotropic subspace of $H^{1}(G, \mathbb{C})$ for $\cup_{G}$ if and only if $h^{*}(E)$ is a (1-) isotropic subspace of $H^{1}(X, \mathbb{C})$ for $\cup_{X}$. 


\section{The Isotropic Subspace Theorem}

By a fibration we mean a surjective morphism $f: M \rightarrow N$ with connected fibers between two compact complex manifolds $M$ and $N$. Two fibrations $f: M \rightarrow C$ and $f^{\prime}: M \rightarrow C^{\prime}$ over projective curves $C$ and $C^{\prime}$ are said to be equivalent if there is an isomorphism $\phi: C \rightarrow C^{\prime}$ such that $f^{\prime}=\phi \circ f$. We denote by $\mathcal{E}(M)$ the set of equivalence classes of fibrations $f: M \rightarrow C$, with $C$ a projective curve of genus $g \geq 2$.

Let $M$ be a compact Kähler manifold. Beauville's work [2] establishes a bijection between the set $\mathcal{E}(M)$ and the set of irreducible components of the first characteristic variety $V_{1}(M)$ passing through the identity of the algebraic group $\mathbb{T}=\operatorname{Hom}\left(\pi_{1}(M), \mathbb{C}^{*}\right)$. In particular, the set $\mathcal{E}(M)$ must be finite.

The Isotropic Subspace Theorem, due to Catanese [6, Theorem 1.10], establishes a relation between the set of equivalence classes of fibrations of a Kähler manifold $M$ over curves of genus $g \geq 2$, and the maximal isotropic subspaces in $H^{1}(M, \mathbb{C})$.

Theorem 3.1 (Catanese [6]). Let $M$ be a compact Kähler manifold. Then, for any maximal isotropic subspace $E \subset H^{1}(M, \mathbb{C})$ of dimension $g \geq 2$, there is a fibration $f: M \rightarrow C$ onto a smooth curve of genus $g$ and a maximal isotropic subspace $E^{\prime} \subset H^{1}(C, \mathbb{C})$ such that $E=f^{*} E^{\prime}$.

For more information on this correspondence, see [7].

\section{The first resonance variety of a Kähler manifold}

Theorem 4.1. Let $M$ be a compact Kähler manifold with $b_{1}(M) \neq 0$. If $R_{1}(M)=$ $H^{1}(M, \mathbb{C})$, then $H^{1}(M, \mathbb{C})$ is 1-isotropic.

Proof. By Hodge theory, we must have $b_{1}(M) \geq 2$. The equality $R_{1}(M)=H^{1}(M, \mathbb{C})$ says that, for any non-zero cohomology class $x \in H^{1}(M, \mathbb{C})$, there is a class $y \in$ $H^{1}(M, \mathbb{C}) \backslash \mathbb{C} \cdot x$ such that $x \cup y=0$. Consequently, the vector space spanned by $x$ and $y$ is a (2-dimensional) isotropic subspace containing $x$.

Let $U_{x}$ be a maximal isotropic subspace of $H^{1}(M, \mathbb{C})$ containing $x$; we must then have $\operatorname{dim} U_{x} \geq 2$. Thus, by Theorem 3.1, there is a fibration $f_{x}: M \rightarrow C_{x}$ onto a smooth projective curve $C_{x}$ of genus $g_{x}=\operatorname{dim} U_{x}$, with $x \in f_{x}^{*}\left(H^{1}\left(C_{x}, \mathbb{C}\right)\right)$.

Recall now that the set $\mathcal{E}(M)$ of equivalence classes of fibrations of $M$ over curves of genus at least 2 is finite. Thus, we may write the first cohomology group of $M$ as a finite union of linear subspaces,

$$
H^{1}(M, \mathbb{C})=\bigcup_{[f] \in \mathcal{E}(M)} f^{*}\left(H^{1}\left(C_{f}, \mathbb{C}\right)\right),
$$

where $f=f_{x}$ for some $x \in H^{1}(M, \mathbb{C})$, and $C_{f}:=C_{x}$. This is possible only if there is a fibration $f_{1}: M \rightarrow C_{1}$ such that $H^{1}(M, \mathbb{C})=f_{1}^{*}\left(H^{1}\left(C_{1}, \mathbb{C}\right)\right)$.

Since $f_{1}$ is a fibration, the induced morphism $f_{1}^{*}: H^{1}\left(C_{1}, \mathbb{C}\right) \rightarrow H^{1}(M, \mathbb{C})$ is injective. The defining property of $f_{1}$ implies that $f_{1}^{*}: H^{1}\left(C_{1}, \mathbb{C}\right) \rightarrow H^{1}(M, \mathbb{C})$ is an isomorphism. 
On the other hand, the induced morphism $f_{1}^{*}: H^{2}\left(C_{1}, \mathbb{C}\right) \rightarrow H^{2}(M, \mathbb{C})$ is also injective. To prove this claim, first note that any cohomology class in $H^{1}(M, \mathbb{C})$ is primitive. Using the Hodge-Riemann bilinear relations (see e.g. [16, p. 123]), it follows that, for any non-zero $(1,0)$-class $\alpha \in H^{1}(M, \mathbb{C})$, the product $\beta=\sqrt{-1} \alpha \cup \bar{\alpha}$ is a non-zero, real, $(1,1)$-class in $H^{2}(M, \mathbb{C})$. Since $f_{1}^{*}: H^{1}\left(C_{1}, \mathbb{C}\right) \rightarrow H^{1}(M, \mathbb{C})$ is an isomorphism, there is an element $a \in H^{1}\left(C_{1}, \mathbb{C}\right)$ such that $f_{1}^{*}(a)=\alpha$. Hence, $f_{1}^{*}(\sqrt{-1} a \wedge \bar{a})=\beta$, and the claim is proved.

Consider now the commuting diagram

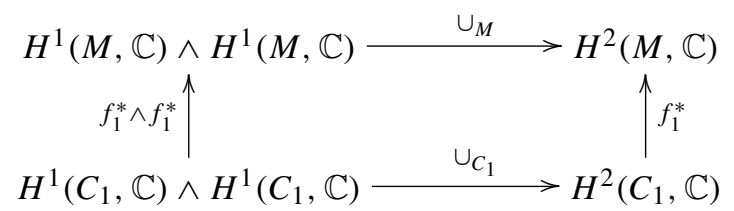

As we saw above, the left arrow is an isomorphism, and the right one is an injection. Since $\cup_{C_{1}}$ surjects onto $H^{2}\left(C_{1}, \mathbb{C}\right)=\mathbb{C}$, we conclude that $\cup_{M}$ has 1-dimensional image.

Remark 4.2. An alternative way to prove Theorem 4.1 is by using the much more general Theorem $C$ from [12], which guarantees that every positive-dimensional component of $R_{1}(M)$ is an 1-isotropic subspace of $H^{1}(M, \mathbb{C})$. This is the argument we had in an earlier version of this paper; at the urging of one of the referees, we came up with the above, more self-contained proof.

\section{The first resonance variety of a 3-manifold}

Let $M$ be a compact, connected, orientable 3-manifold. Fix an orientation on $M$, that is, pick a generator $[M] \in H^{3}(M, \mathbb{Z}) \cong \mathbb{Z}$. With this choice, the cup-product on $M$ determines an alternating 3-form $\mu=\mu_{M}$ on $H^{1}(M, \mathbb{Z})$, given by

$$
\mu(x, y, z)=\langle x \cup y \cup z,[M]\rangle,
$$

where $\langle$,$\rangle is the Kronecker pairing. In turn, the cup-product map \cup_{M}: H^{1}(M, \mathbb{Z}) \wedge$ $H^{1}(M, \mathbb{Z}) \rightarrow H^{2}(M, \mathbb{Z})$ is determined by $\mu$, via $\langle x \cup y, \gamma\rangle=\mu(x, y, z)$, where $z=$ $\operatorname{PD}(\gamma)$ is the Poincaré dual of $\gamma \in H_{2}(M, \mathbb{Z})$.

Now fix a basis $\left\{e_{1}, \ldots, e_{n}\right\}$ for $H^{1}(M, \mathbb{C})$, and choose as basis for $H^{2}(M, \mathbb{C})$ the set $\left\{e_{1}^{\vee}, \ldots, e_{n}^{\vee}\right\}$, where $e_{i}^{\vee}$ denotes the Kronecker dual of the Poincaré dual of $e_{i}$. Then

$$
\mu\left(e_{i}, e_{j}, e_{k}\right)=\left\langle\sum_{1 \leq m \leq n} \mu_{i, j, m} e_{m}^{\vee}, \operatorname{PD}\left(e_{k}\right)\right\rangle=\mu_{i, j, k} .
$$

Recall from (3) the $n \times n$ matrix with entries $\Delta_{k, j}=\sum_{i=1}^{n} \mu_{i, j, k} x_{i}$. Since $\mu$ is an alternating form, $\Delta$ is a skew-symmetric matrix.

Proposition 5.1. Let $M$ be a closed, orientable 3-manifold. Then:

(1) $H^{1}(M, \mathbb{C})$ is not 1 -isotropic.

(2) If $b_{1}(M)$ is even, then $R_{1}(M)=H^{1}(M, \mathbb{C})$. 
Proof. To prove (1), suppose $\operatorname{dim} \operatorname{im}\left(\cup_{M}\right)=1$. This means there is a hyperplane $E \subset$ $H:=H^{1}(M, \mathbb{C})$ such that $x \cup y \cup z=0$ for all $x, y \in H$ and $z \in E$. Hence, the skew 3-form $\mu: \bigwedge^{3} H \rightarrow \mathbb{C}$ factors through a skew 3-form $\bar{\mu}: \bigwedge^{3}(H / E) \rightarrow \mathbb{C}$. But $\operatorname{dim} H / E=1$ forces $\bar{\mu}=0$, and so $\mu=0$, a contradiction.

To prove (2), recall $R_{1}(M)=V\left(E_{1}(\Delta)\right)$. Since $\Delta$ is a skew-symmetric matrix of even size, it follows from Buchsbaum-Eisenbud [4, Corollary 2.6] that $V\left(E_{1}(\Delta)\right)=$ $V\left(E_{0}(\Delta)\right)$ (see [8, eq. (6.9)]). But $\Delta \cdot \vec{x}=0$ implies det $\Delta=0$, and so $V\left(E_{0}(\Delta)\right)=H$.

Remark 5.2. As noted by S. Papadima, the following holds. Suppose $M$ is a closed, orientable 3-manifold, with $b_{1}(M)$ odd. Then $R_{1}(M) \neq H^{1}(M, \mathbb{C})$ if and only if $\mu_{M}$ is generic, in the sense of [3].

\section{Kazhdan's property $T$}

The following question is due to J. Carlson and D. Toledo (see J. Kollár [22]): For a Kähler group $G$, is $b_{2}(G) \neq 0$ ? This question was answered in the affirmative by A. Reznikov in [28], under an additional assumption, as follows.

Theorem 6.1 (Reznikov [28]). Let $G$ be a Kähler group. If $G$ does not satisfy Kazhdan's property $T$, then $b_{2}(G) \neq 0$.

Recall that a discrete group $G$ satisfies Kazhdan's property $T$ (for short, $G$ is a Kazhdan group) if and only if $H^{1}(G, \mathcal{H})=0$ for all orthogonal or unitary representations of $G$ on a Hilbert space $\mathcal{H}$ (see de la Harpe and Valette [18, p. 47]). In particular, if $b_{1}(G) \neq 0$, then $G$ is not Kazhdan. (For a simple proof of Theorem 6.1] in this case, see [21].)

We will also need the following relationship between 3-manifold groups and Kazhdan's property $T$, established by K. Fujiwara in [13].

Theorem 6.2 (Fujiwara [13]). Let $G$ be the fundamental group of a closed, orientable 3-manifold. If $G$ satisfies Kazhdan's property $T$, then $G$ is finite.

In fact, the theorem is valid for any subgroup $G<\pi_{1}(M)$, where $M$ is a compact (not necessarily boundaryless), connected, orientable 3-manifold. Fujiwara further assumes that each piece of the canonical decomposition of $M$ along embedded spheres, disks and tori admits one of the eight geometric structures in the sense of Thurston, but this is now guaranteed by the work of Perelman [26, 27].

\section{Kähler 3-manifold groups}

We are now in a position to prove Theorem 1.1 from the introduction.

Let $G$ be the fundamental group of a compact, connected 3-manifold $M$. Suppose $G$ is a Kähler group, and $G$ is not finite. 
Step 1. A finite-index subgroup of a Kähler group is again a Kähler group (see [1, Example 1.10]). Passing to the orientation double cover of $M$ if necessary, we may as well assume $M$ is orientable.

Step 2. Since $G$ is an infinite, orientable 3-manifold group, $G$ is not Kazhdan, by $\mathrm{Fu}$ jiwara's Theorem 6.2. Since $G$ is Kähler and not Kazhdan, $b_{2}(G) \neq 0$, by Reznikov's Theorem6.1.

Step 3. Since $b_{2}(M) \geq b_{2}(G)$, we must also have $b_{2}(M) \neq 0$. By Poincaré duality, $b_{1}(M)=b_{2}(M)$. Hence, $b_{1}(G)=b_{1}(M)$ is not zero.

Step 4. Since $G$ is Kähler, $b_{1}(G)$ must be even. Since $M$ is a closed, orientable 3manifold with $G=\pi_{1}(M)$, Proposition 5.1 tells us that $R_{1}(G)=H^{1}(G, \mathbb{C})$ and $H^{1}(G, \mathbb{C})$ is not 1-isotropic. Since, on the other hand, $G$ is Kähler, Theorem 4.1 tells us that $b_{1}(G)=0$.

Our assumptions have led us to a contradiction. Thus, the theorem is proved.

Acknowledgments. This work was done during the second author's visit at Université de NiceSophia Antipolis in September, 2007. He thanks the Laboratoire Jean A. Dieudonné for its support and hospitality during his stay in Nice. Both authors thank the referees for constructive comments and helpful suggestions that led to improvements in the presentation and content of the paper.

\section{References}

[1] Amorós, J., Burger, M., Corlette, K., Kotschick, D., Toledo, D.: Fundamental Groups of Compact Kähler Manifolds. Math. Surveys Monogr. 44, Amer. Math. Soc., Providence, RI (1996) Zbl 0849.32006 MR 1379330

[2] Beauville, A.: Annulation du $H^{1}$ pour les fibrés en droites plats. In: Complex Algebraic Varieties (Bayreuth, 1990), Lecture Notes in Math. 1507, Springer, Berlin, 1-15 (1992) Zbl 0792.14006 MR 1178716

[3] Berceanu, B., Papadima, S.: Cohomologically generic 2-complexes and 3-dimensional Poincaré complexes. Math. Ann. 298, 457-480 (1994) Zbl 0791.57007 MR 1262770

[4] Buchsbaum, D., Eisenbud, D.: Algebra structures for finite free resolutions, and some structure theorems for ideals of codimension 3. Amer. J. Math. 99, 447-485 (1977) Zbl 0373.13006 MR 0453723

[5] Campana, F.: Ensembles de Green-Lazarsfeld et quotients resolubles des groupes de Kähler. J. Algebraic Geom. 10, 599-622 (2001) Zbl 1072.14512 MR 1838973

[6] Catanese, F.: Moduli and classification of irregular Kaehler manifolds (and algebraic varieties) with Albanese general type fibrations. Invent. Math. 104, 263-289 (1991) Zbl 0743.32025 MR 1098610

[7] Catanese, F.: Fibred surfaces, varieties isogenous to a product and related moduli spaces. Amer. J. Math. 122, 1-44 (2000) Zbl 0983.14013 MR 1737256

[8] Cohen, D., Suciu, A.: Boundary manifolds of projective hypersurfaces. Adv. Math. 206, 538566 (2006) Zbl 1110.14036 MR 2263714

[9] Deligne, P., Griffiths, P., Morgan, J., Sullivan, D.: Real homotopy theory of Kähler manifolds. Invent. Math. 29, 245-274 (1975) Zbl 0312.55011 MR 0382702

[10] Delzant, T.: Trees, valuations, and the Green-Lazarsfeld sets. Geom. Funct. Anal. 18, 12361250 (2008) Zbl pre05508775 MR 2465689 
[11] Delzant, T., Gromov, M.: Cuts in Kähler groups. In: Infinite Groups: Geometric, Combinatorial and Dynamical Aspects, Progr. Math. 248, Birkhäuser, Basel, 31-55 (2005) Zbl 1116.32016 MR 2195452

[12] Dimca, A., Papadima, S., Suciu, A.: Topology and geometry of cohomology jump loci. Duke Math. J. 148 (2009), to appear; arXiv:0902.1250

[13] Fujiwara, K.: 3-manifold groups and property $T$ of Kazhdan. Proc. Japan Acad. Ser. A Math. Sci. 75, 103-104 (1999) Zbl 0957.57004 MR 1729853

[14] Gompf, R.: A new construction of symplectic 4-manifolds. Ann. of Math. 142, 527-595 (1995) Zbl 0849.53027 MR 1356781

[15] Green, M., Lazarsfeld, R.: Higher obstructions to deforming cohomology groups of line bundles. J. Amer. Math. Soc. 4, 87-103 (1991) Zbl 0735.14004 MR 1076513

[16] Griffiths, P., Harris, J.: Principles of Algebraic Geometry. Wiley, New York (1978) Zbl 0408.14001 MR 0507725

[17] Gromov, M.: Sur le groupe fondamental d'une variété kählérienne. C. R. Acad. Sci. Paris Sér. I 308, 67-70 (1989) Zbl 0661.53049 MR 0983460

[18] de la Harpe, P., Valette, A.: La propriété $(T)$ de Kazhdan pour les groupes localement compacts. Astérisque 175 (1989) Zbl 0759.22001 MR 1023471

[19] Hernández-Lamoneda, L.: Non-positively curved 3-manifolds with non-Kähler $\pi_{1}$. C. R. Acad. Sci. Paris Sér. I Math. 332, 249-252 (2001) Zbl 0992.53028 MR 1817371

[20] Jaco, W.: Lectures on Three-Manifold Topology. CBMS Reg. Conf. Ser. Math. 43, Amer. Math. Soc., Providence, RI (1980) Zbl 0433.57001 MR 0565450

[21] Johnson, F. E. A., Rees, E.: On the fundamental group of a complex algebraic manifold. Bull. London Math. Soc. 19, 463-466 (1987) Zbl 0608.53061 MR 0898726

[22] Kollár, J.: Shafarevich Maps and Automorphic Forms. Princeton Univ. Press, Princeton, NJ (1995) Zbl 0871.14015 MR 1341589

[23] Milnor, J.: Groups which act on $S^{n}$ without fixed points. Amer. J. Math. 79, 623-630 (1957) Zbl 0078.16304 MR 0090056

[24] Morgan, J., Tian, G.: Ricci Flow and the Poincaré Conjecture. Clay Math. Monogr. 3, Amer. Math. Soc., Providence, RI, and Clay Math. Inst., Cambridge, MA (2007) Zbl pre05188193 MR 2334563

[25] Napier, T., Ramachandran, M.: Filtered ends, proper holomorphic mappings of Kähler manifolds to Riemann surfaces, and Kähler groups. Geom. Funct. Anal. 17, 1621-1654 (2008) Zbl 1144.32017 MR 2377498

[26] Perelman, G.: Ricci flow with surgery on three-manifolds. arXiv:math.DG/0303109 Zbl 1130.53002

[27] Perelman, G.: Finite extinction time for the solutions to the Ricci flow on certain threemanifolds. arXiv:math.DG/0307245 Zbl 1130.53003

[28] Reznikov, A., The structure of Kähler groups. I. Second cohomology. In: Motives, Polylogarithms and Hodge Theory, Part II (Irvine, CA, 1998), Int. Press Lect. Ser. 3, II, Int. Press, Somerville, MA, 717-730 (2002) Zbl 1048.32008 MR 1978716

[29] Serre, J.-P.: Sur la topologie des variétés algébriques en charactéristique $p$. In: Symposium internacional de topología algebraica (Mexico City, 1958), UNAM, 24-53 (1958) Zbl 0098.13103 MR 0098097

[30] Simpson, C.: Higgs bundles and local systems. Inst. Hautes Études Sci. Publ. Math. 75, 5-95 (1992) Zbl 0814.32003 MR 1179076

[31] Simpson, C.: The construction problem in Kähler geometry. In: Different Faces of Geometry, Int. Math. Ser. 3, Kluwer/Plenum, New York, 365-402 (2004) Zbl 1064.32013 MR 2103668

[32] Taubes, C. H.: The existence of anti-self-dual conformal structures. J. Differential Geom. 36, 163-253 (1992) Zbl 0822.53006 MR 1168984 